\title{
Role of electromagnetic trapped modes in extraordinary transmission in nanostructured materials
}

\author{
A. G. Borisov, ${ }^{1,2, *}$ F. J. García de Abajo, ${ }^{2,3}$ and S. V. Shabanov $v^{4,2}$ \\ ${ }^{1}$ Laboratoire des Collisions Atomiques et Moléculaires, UMR CNRS-Université Paris-Sud 8625, Bât. 351, \\ Université Paris-Sud, 91405 Orsay CEDEX, France \\ ${ }^{2}$ Donostia International Physics Center (DIPC), Apartado Postal 1072, 20080 San Sebastián, Spain \\ ${ }^{3}$ Centro Mixto CSIC-UPV/EHU, Apartado Postal 1072, 20080 San Sebastián, Spain \\ ${ }^{4}$ Department of Mathematics, University of Florida, Gainesville, Florida 23611, USA \\ (Received 5 October 2004; revised manuscript received 3 December 2004; published 18 February 2005)
}

\begin{abstract}
We assert that the physics underlying the extraordinary light transmission (reflection) in nanostructured materials can be understood from rather general principles based on the formal scattering theory developed in quantum mechanics. The Maxwell equations in passive (dispersive and absorptive) linear media are written in the form of the Schrödinger equation to which the quantum mechanical resonant scattering theory (the Lippmann-Schwinger formalism) is applied. It is demonstrated that the existence of long-lived quasistationary eigenstates of the effective Hamiltonian for the Maxwell theory naturally explains the extraordinary transmission properties observed in various nanostructured materials. Such states correspond to quasistationary electromagnetic modes trapped in the scattering structure. Our general approach is also illustrated with an example of the zero-order transmission of the TE-polarized light through a metal-dielectric grating structure. Here a direct on-the-grid solution of the time-dependent Maxwell equations demonstrates the significance of resonances (or trapped modes) for extraordinary light transmission.
\end{abstract}

DOI: 10.1103/PhysRevB.71.075408

PACS number(s): 42.79.Dj, 41.20.Jb, 42.25.Fx, 03.65.Nk

\section{INTRODUCTION}

Supported by technological progress, studies of the interaction of electromagnetic radiation with nanostructured materials have become an area of intense research driven by potential applications in optics and photonics. ${ }^{1-4}$ In particular, it has been found that metal ${ }^{3-16}$ and dielectric ${ }^{17-24}$ grating structures possess extraordinary transmission (reflection) properties within narrow intervals of wavelengths close to the grating period. While for dielectric gratings a common point of view on this phenomenon, as occurring due to the existence of trapped modes or guided wave resonances, ${ }^{18-24}$ seems to be established, there is still an ongoing discussion on the origin of a nearly $100 \%$ light transmission within narrow wavelength range(s) observed in slit and hole arrays made in metal films. Although similar results are obtained with different theoretical approaches, in which the Maxwell equations are numerically solved, an explanation of the underlying physics, as due to excitations of coupled surface plasmons and/or cavity modes, ${ }^{5,6}$ competes with the dynamical diffraction theory point of view. ${ }^{9,10}$ Remarkably, all available studies of metal grating structures have been carried out with the TM-polarized light (the magnetic field is parallel to the slits) where surface plasmons can indeed be excited.

Transmission and reflection properties of grating structures are typically studied by stationary methods in the frequency domain. Nevertheless, the dynamics of light scattering on gratings can partly be guessed from stationary results. Indeed, consider a femtosecond (broadband) pulse impinging on a grating structure such that the spectral range of the pulse is much larger than the grating transmission window(s). From the uncertainty principle it follows that, in order for transmission of light to occur only within a narrow frequency range, the radiation should last much longer than the duration of the initial pulse. This implies that the corresponding electromagnetic modes have to be trapped by the nanostructured material during a sufficiently long time. Such modes are known in scattering theory as scattering resonances. ${ }^{25}$

Here we propose a general point of view on the physics underlying the extraordinary light transmission in nanostructured materials. By reformulating the Maxwell equations in the form of the Schrödinger equation and by using quantum scattering theory, we show that this phenomenon is a direct consequence of the existence of trapped electromagnetic modes possessing large lifetimes. Based on this general principle, various structures can be custom designed that would transmit or reflect light within a designated narrow wavelength range(s). As an example to illustrate our approach, transmission properties of a metal-dielectric grating structure are studied for TE-polarized light (the electric field is parallel to the slits) impinging normally on the grating. A direct onthe-grid solution of the time-dependent Maxwell equations demonstrates the significance of resonances (or trapped modes) for the enhanced light transmission. Interestingly enough, such a system has never been studied before, probably because of the absence of the coupling of electromagnetic modes to plasmons, often thought to be the main mechanism of the extraordinary light transmission. It should be understood that our choice of the geometry does not imply an attempt to favor either the plasmon- or cavity-mode point of view in, generally, strongly coupled systems.

\section{METAL-DIELECTRIC GRATING}

We begin with an example of a grating structure sketched in Fig. 1 to illustrate our basic idea. The grating structure has 


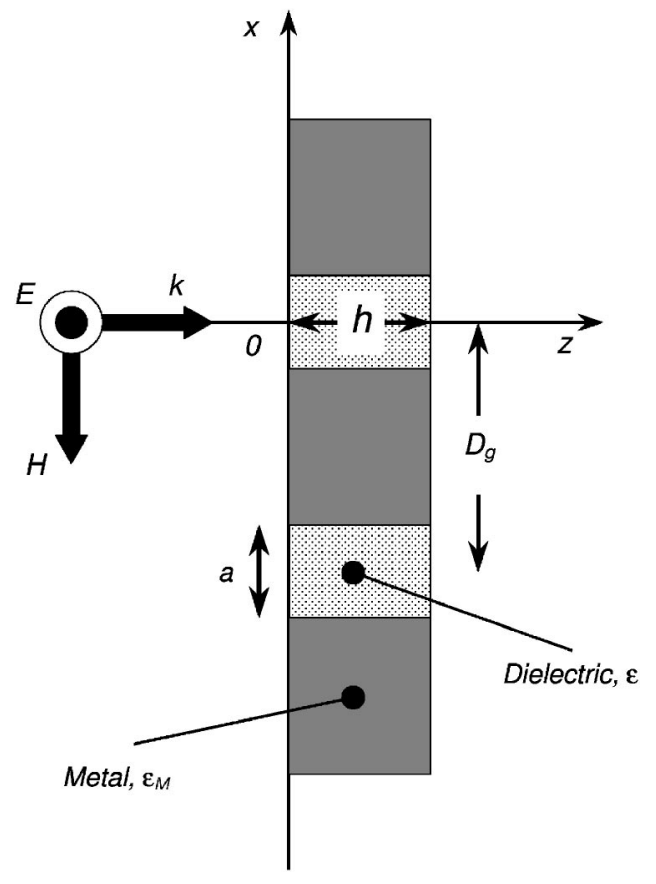

FIG. 1. Schematic representation of the studied system. The radiation is incident along the normal to the slab (the $z$ axis), consisting of a grating structure with alternating regions of metal and dielectric along the $x$ direction. The dark grey and shaded regions correspond, respectively, to the metal and dielectric parts of the structure. The system is translationally invariant along the $y$ direction.

translational symmetry along one of the Euclidean axes, chosen to be the $y$ axis. The structure is periodic along the $x$ axis with period $D_{g}$, and the $z$ direction is transverse to the structure. For the sake of comparison with previous works, ${ }^{5}$ the parameters are chosen to be: the grating period $D_{g}$ $=1.75 \mu \mathrm{m}$, the thickness (along the $z$ axis) $h=0.8-1.4 \mu \mathrm{m}$, and the opening width $a=0.3-0.35 \mu \mathrm{m}$. In the case of the TM radiation impinging on the grating with no dielectric fillings, the extraordinary transmission properties have already been reported and analyzed..$^{5,7,10,11,13}$ The question arises whether enhanced transmission can be obtained for the TE radiation or, according to our remarks in the previous section, whether or not trapped modes exist and can be excited.

To obtain a rough estimate of the wavelengths of possible trapped (or quasistationary) electromagnetic modes in the system, consider first the case of a perfect metal grating. For a moment, we also neglect the effects due to a finite thickness of the grated metal slab in the $z$ direction. In other words, we neglect the coupling between trapped modes and radiation modes outside the grating. Then the $x$ component of the wave vector is quantized as a consequence of the zero boundary conditions at the metal-dielectric interface. A quantization of the $z$ component of the wave vector can be understood as Fabry-Perot modes in a dielectric slab inside the metallic waveguide-i.e., the modes for which the dielectric slab is $100 \%$ transparent. Admissible (quantized) values of the wave vector inside the grating define wavelengths $\lambda_{n m}$ of trapped electromagnetic modes that can be excited by the incident radiation

$$
\varepsilon\left(\frac{2}{\lambda_{n m}}\right)^{2}=\left(\frac{n}{a}\right)^{2}+\left(\frac{m}{h}\right)^{2}
$$

where $\varepsilon$ is the dielectric constant, and $n, m=1,2, \ldots$. Note that, since $a<h$ for the grating geometry, the first term on the right-hand-side (RHS) of Eq. (1) is the most relevant for the threshold to excite quasistationary states (standing waves). In what follows we are interested in the zerodiffraction mode for wavelengths $\lambda \geqslant D_{g}$ so that the reflected and transmitted beams propagate along the $z$ axis. In this case the threshold for trapped modes to exist is determined by the lowest mode, $n=m=1$, leading to the condition

$$
\varepsilon \geqslant \frac{1}{4}\left[\left(\frac{D_{g}}{a}\right)^{2}+\left(\frac{D_{g}}{h}\right)^{2}\right] .
$$

Taking into account the grating parameters, condition (2) can be satisfied only if $\varepsilon \geqslant 9.7$ for the range of $a$ and $h$ specified above. Thus, if there is no dielectric filling $(\varepsilon=1)$, the grating structure would totally reflect the TE radiation with $\lambda \geqslant D_{g}$, which is indeed supported by our numerical simulations. This is in contrast to the TM radiation case where $100 \%$ transmission can be reached for the very same grating with no dielectric fillings and made of the perfect conductor. ${ }^{5}$

In our numerical study, we consider the grating with openings filled with a nondispersive dielectric with $\varepsilon=11.9$ (this corresponds to $\mathrm{Si}$ within the wavelength range under consideration). The metal is described by the Drude model:

$$
\varepsilon_{M}(\omega)=1-\frac{\omega_{p}^{2}}{\omega^{2}+i \omega \gamma} .
$$

For the sake of comparison with previous works the plasma frequency and the attenuation are taken as $\omega_{p}=9 \mathrm{eV}$ and $\gamma$ $=0.1 \mathrm{eV}$ (Ref. 5). Transmission and reflection properties of the grating structure are calculated by means of the wave packet propagation method. The method is based on the representation of the Maxwell equations in the form of the Schrödinger equation for which the initial value problem is numerically solved by a time-stepping algorithm. A detailed description can be found elsewhere. ${ }^{23,24}$

For the specific case considered here, the Hamiltonian formalism is as follows. Let $\mathbf{D}=\mathbf{E}+\mathbf{P}$ where $\mathbf{D}, \mathbf{E}$, and $\mathbf{P}$ are the electric induction, the electric field, and the medium polarization vector, respectively. In the Drude model the medium polarization vector satisfies the second-order differential equation

$$
\ddot{\mathbf{P}}+\gamma \dot{\mathbf{P}}=\omega_{p}^{2} \mathbf{E}
$$

where derivatives with respect to time $t$ are denoted by overdots. Equation (4) must be solved with zero initial conditions, $\mathbf{P}=\dot{\mathbf{P}}=0$ at $t=0$. Define an auxiliary field $\mathbf{Q}$ by $\dot{\mathbf{P}}$ $=\omega_{p} \mathbf{Q}$. The Maxwell's equations are cast in the Schrödinger form:

$$
i \dot{\Psi}=H \Psi
$$

where the wave function $\Psi$ and the Hamiltonian $H$ are 


$$
\Psi=\left(\begin{array}{c}
\sqrt{\varepsilon} \mathbf{E} \\
\mathbf{B} \\
\mathbf{Q}
\end{array}\right), \quad H=\left(\begin{array}{ccc}
0 & i c \varepsilon^{-1 / 2} \boldsymbol{\nabla} \times & -i \omega_{p} \\
-i c \boldsymbol{\nabla} \times \varepsilon^{-1 / 2} & 0 & 0 \\
i \omega_{p} & 0 & -i \gamma
\end{array}\right)
$$

The norm of the wave function, $\|\Psi\|^{2}=\int d \mathbf{r}^{\dagger} \Psi$, is proportional to the total electromagnetic energy of the wave packet. ${ }^{23,26}$ When the attenuation is not present, $\gamma=0$, the Hamiltonian is Hermitian and the norm (energy) is conserved. The quantities $\varepsilon$ and $\omega_{p}$ are position dependent so that $\varepsilon=1$ everywhere outside the dielectric inclusions and $\omega_{p}=0$ everywhere outside the metal part of the grating.

It follows from Eq. (5) that $\Psi(t+\Delta t)=\exp (-i \Delta t H) \Psi(t)$. In our simulations, the action of the infinitesimal evolution operator $\exp (-i \Delta t H)$ on the wave function $\Psi$ is carried out by the algorithm described in Ref. 23. The initial wave packet is Gaussian and propagates along the $z$ axis perpendicular to the grating. Its spectrum is broad enough to cover the frequency range of interest. A change of variables is used to enhance the sampling efficiency in the vicinity of medium interfaces so that the boundary conditions are accurately reproduced by the Fourier-grid pseudospectral method. ${ }^{27,28} \mathrm{~A}$ typical size of the mesh corresponds to $-15 D_{g} \leqslant z \leqslant 15 D_{g}$ and $-0.5 D_{g} \leqslant x \leqslant 0.5 D_{g}$ with 512 and 128 knots, respectively. The frequency resolved transmission and reflection coefficients are obtained via the time-to-frequency Fourier transform of the signal at some distance in front and behind the grating. ${ }^{29}$ An absorbing layer is introduced at the grid boundaries in order to suppress artificial reflections of the wave packet. ${ }^{30}$

\section{RESULTS AND DISCUSSION}

In Fig. 2(a) we show an interpolated image of the time evolution of the electric field $E_{y}$ along the $z$ axis passing through the center of the grating (see Fig. 1). The grating structure is characterized by $\varepsilon=11.9, D_{g}=1.75 \mu \mathrm{m}, a$ $=0.35 \mu \mathrm{m}$, and $h=1.4 \mu \mathrm{m}$. The red and blue colors correspond to positive and negative values of the field, respectively. The horizontal axis represents the $z$ coordinate expressed in units of the grating period, $D_{g}$. The grating extends from $z=0$ to $z=h / D_{g}$. The vertical axis represents the propagation time measured in femtoseconds. The initial pulse impinging on the grating has a duration of approximately $25 \mathrm{fs}$. The instant when the pulse hits the grating followed by the main reflected signal is clearly visible in the figure. One also observes that a fraction of the electromagnetic energy is stored in the grating structure and leads to a long-lasting radiation on both the transmission and reflection sides. This lasing effect extends to a picosecond time range; i.e., it is much longer than the duration of the initial pulse. It can be explained as due to the existence of trapped electromagnetic modes or resonances. It is the radiation of decaying trapped modes that comes with a phase opposite to the corresponding harmonic in the initially reflected pulse to the left from the grating structure and leads finally to the reduced reflection. The same lasing effect to the right from the grating structure is responsible for high transmission at the same
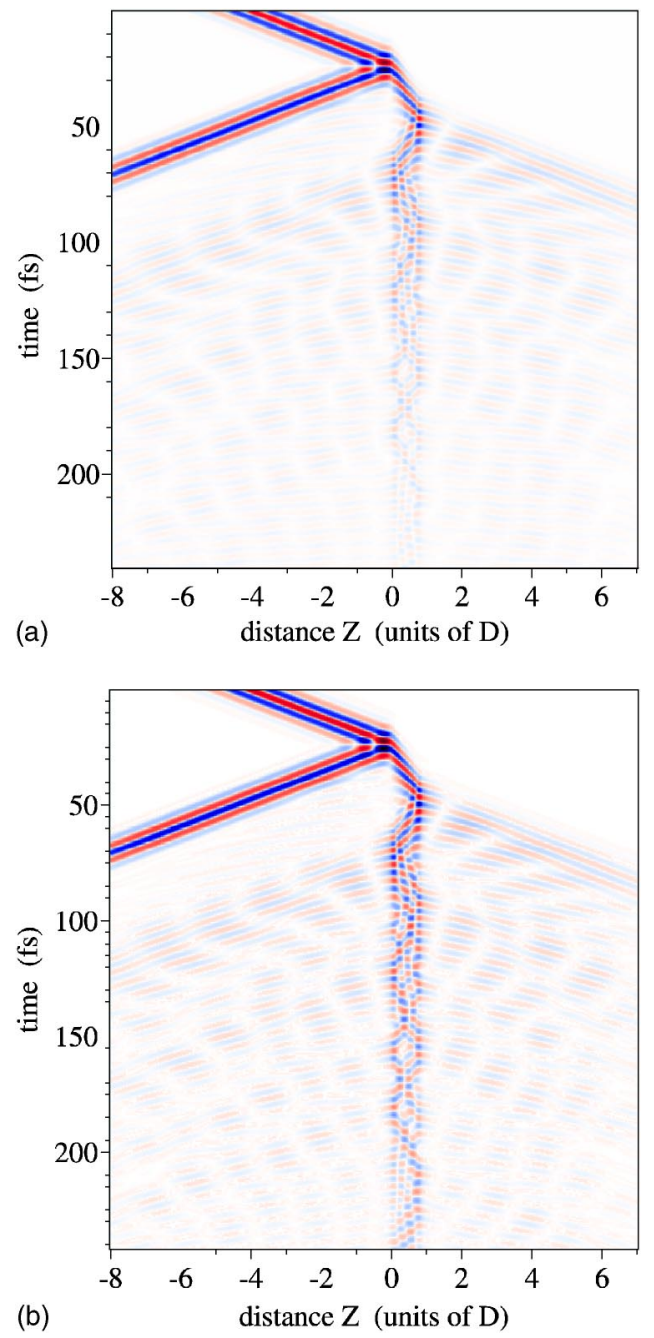

FIG. 2. (Color online) (a) Interpolated image of the time evolution of the electric field $E_{y}$ along the $z$ axis passing through the center of a dielectric region of the grating (see Fig. 1). The grating structure is characterized by $\varepsilon=11.9, D_{g}=1.75 \mu \mathrm{m}, a=0.35 \mu \mathrm{m}$, and $h=1.4 \mu \mathrm{m}$. The red and blue colors correspond, respectively, to positive and negative values of the field with the color intensity related to the field magnitude. The horizontal axis represents the $z$ coordinate expressed in units of the grating period, $D_{g}$. The grating extends from $z=0$ to $z=h / D_{g}$. The vertical axis represents the propagation time measured in femto-seconds. The metal is described by the dielectric function of Eq. (3). (b) The same as (a), but with no damping inside the metal $(\gamma=0)$.

frequency (see further discussion and results in Fig. 3).

Figure 2(b) shows the dynamics of the electric field $E_{y}$ in the same setting, but the attenuation $\gamma$ is set to zero. In this case, the trapped modes do not dissipate their energy into the metal. As a result, they live longer, which is clearly seen from comparison of the color intensity of the vertical strip in the middle of Figs. 2(a) and 2(b) (which represents the electric field of the trapped modes).

The calculated transmission coefficient is presented in Fig. 3 as a function of the wavelength expressed in units of the grating period, $D_{g}$. Each of the resonances observed in Fig. 3 is associated with the corresponding trapped mode. The rough estimate given in Eq. (1) of their energies (fre- 


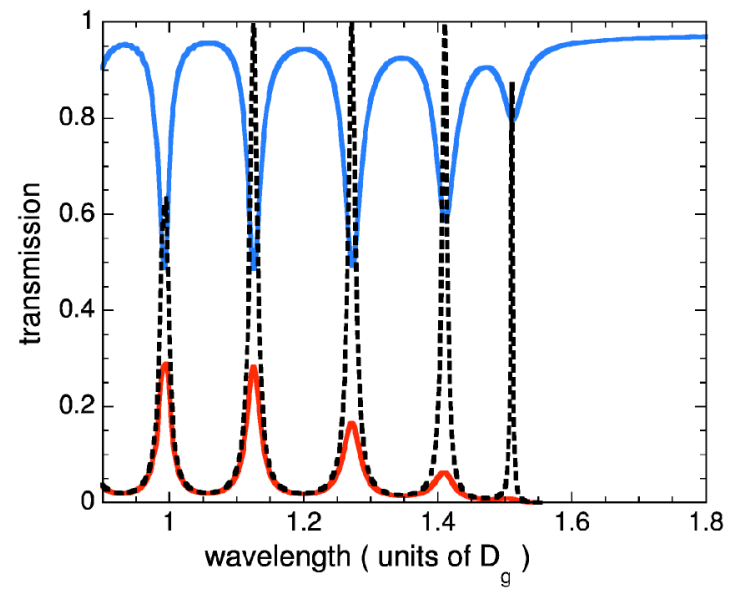

FIG. 3. (Color online) Zero-order transmission coefficient as a function of the wavelength of the incident radiation measured in units of the period, $D_{g}$. The calculation is carried out for the grating structure characterized by $\varepsilon=11.9, D_{g}=1.75 \mu \mathrm{m}, a=0.35 \mu \mathrm{m}$, and $h=1.4 \mu \mathrm{m}$. The solid red and dashed black curves correspond to calculations done with $(\gamma=0.1 \mathrm{eV})$ and without $(\gamma=0)$ damping in the metal, respectively. The sum of the reflection and transmission coefficients for $\gamma=0.1 \mathrm{eV}$ is shown as the blue curve. Its deviation from 1 represents the loss of electromagnetic energy because of the absorption in the metal.

quencies) can be improved by taking into account the penetration of the field into the metal whose dielectric properties are described by Eq. (3). This yields

$$
\omega_{m}=\sqrt{\Omega^{2}+\left(\frac{\pi c m}{h \sqrt{\varepsilon}}\right)^{2}}, \quad m=1,2, \ldots,
$$

where $\Omega$ is the frequency of the lowest eigenmode in the stationary equation

$$
\frac{\partial^{2} E(x)}{\partial x^{2}}+\frac{\Omega^{2}}{c^{2}} \varepsilon_{M}(x, \Omega) E(x)=0 .
$$

Equation (8) is solved numerically under the condition that $E(x)$ must decay exponentially outside the interval $0<x<a$ (i.e., in the metal). For $a=0.35 \mu \mathrm{m}$ and $h=1.4 \mu \mathrm{m}$, this gives the following resonant wavelengths (expressed in the units of grating period): $\lambda_{1}=1.493, \lambda_{2}=1.352, \lambda_{3}=1.185$, $\lambda_{4}=1.031$, and $\lambda_{5}=0.899$. The improved estimate of the resonant wavelengths agrees closely with the results obtained from numerical simulations for the exact problem only for the largest resonant wavelength $\left(\lambda_{1}=1.493\right)$. The wavelengths corresponding to the maximum of the transmission coefficient for higher modes are redshifted as compared to the estimated values. A similar result was reported by Takakura, ${ }^{7}$ but for the TM incident wave polarization. The redshift can be explained by spreading of the trapped modes into the vacuum due to a finite thickness of the grating (see also Fig. 4 and its discussion below), while the Fabry-Perrot modes of the electromagnetic field, used in our rough estimate, satisfy the zero-boundary condition at the dielectricvacuum interface. Clearly, an increase of the spatial volume occupied by a standing wave implies increasing its wavelength and, hence, lowering its frequency.

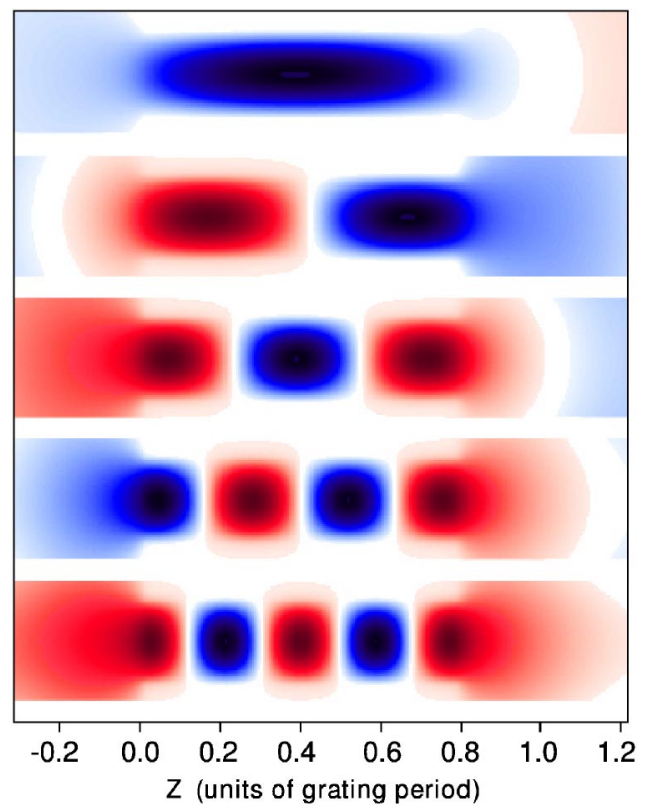

FIG. 4. (Color online) Electric field of the trapped modes inside the grating as function of $x$ and $z$ coordinates. The results are presented for the grating with parameters $\varepsilon=11.9, D_{g}=1.75 \mu \mathrm{m}, a$ $=0.35 \mu \mathrm{m}$, and $h=1.4 \mu \mathrm{m}$. Each colored strip represents the electric field of the trapped mode associated with corresponding maxima of the transmission coefficient (see Fig. 3). The data (from top to bottom) starts with the largest wavelength resonance at $\lambda$ $\approx 1.5 D_{g}$ and ends with the resonance at $\lambda \approx 1 D_{g}$. The red and blue colors correspond, respectively, to positive and negative values of the field. The data has been normalized to 1 at maximum so that color scale covers the range $[-1,+1]$. The $x$ range for each strip (along the vertical axis) corresponds to $-0.26 \mu \mathrm{m} \leqslant x \leqslant 0.26 \mu \mathrm{m}$ and the range for $z$ (horizontal axis) is specified in the figure in units of $D_{g}$.

Because of dissipative losses of energy in the Drude metal, the transmittance does not reach $100 \%$ and is, in fact relatively small. While for a lossless medium the sum of the reflection and transmission coefficients must be 1 as follows from electromagnetic energy conservation, this is not the case when attenuation is present (blue curve in Fig. 3). The maximal loss of energy corresponds to resonant wavelengths. This can be easily understood because the trapped modes remain in contact with the metal much longer than the main pulse [cf. Figs. 2(a) and 2(b)] and, therefore, can dissipate more energy through exciting surface electrical currents in the metal. We further illustrate this point by computing the transmission coefficient in the same system but without attenuation $(\gamma=0)$. The result is shown by the dashed black curve, which reaches 1 at resonant wavelengths. Observe the deviation of the transmission coefficient from 1 for the narrowest resonance at $\lambda \sim 1.5 D_{g}$ even in the absence of absorption. This resonance possesses an extremely long lifetime, so much so that we had to stop the calculation before it had decayed completely; that is, the total energy trapped into this mode was not completely radiated out and, hence, was not fully accounted for. For the resonance at $\lambda<D_{g}$ the transmission coefficient does not reach 1 because we study only the zero-diffraction-order scattering channel. 
Wave functions (field configurations) of the trapped modes can be extracted from the time-dependent wave packet by the time-to-frequency Fourier transform:

$$
\Psi(\omega)=\int_{0}^{\infty} \Psi(t) e^{i \omega t} d t
$$

The wave function of a particular trapped mode is obtained by setting $\omega$ to the frequency at which the transmission coefficient attains the corresponding maximum. In order to improve the contrast, the wave functions have been extracted for the case with no attenuation. The results are presented in Fig. 4 for the grating with parameters $\varepsilon=11.9, D_{g}$ $=1.75 \mu \mathrm{m}, a=0.35 \mu \mathrm{m}$, and $h=1.4 \mu \mathrm{m}$. There are five colored strips in Fig. 4. Each colored strip represents the electric field of the trapped mode associated with corresponding maxima of the transmission coefficient (see Fig. 3). The data (from the top to the bottom) start with the largest wavelength resonance at $\lambda \approx 1.5 D_{g}$ and end with the resonance at $\lambda$ $\approx 1 D_{g}$. The red and blue colors represent, respectively, negative and positive values of the electric field amplitude. Each colored strip covers the coordinate range: $-0.26 \mu \mathrm{m} \leqslant x$ $\leqslant 0.26 \mu \mathrm{m}$ (along the vertical axis) and the range for $z$ (horizontal axis) is specified in the figure in units of $D_{g}$. The fields localized inside the grating and fields radiated into the vacuum (the lasing effect) are clearly visible in the figure. The trapped modes localized inside the dielectric part of the grating exhibit a nearly Fabry-Perot pattern with respect to the quantization in the $z$ direction. Observe a slight spreading of the field into the vacuum regions, $z<0$ and $z>h$, which explains the redshift of the resonant wavelengths as compared to the pure Fabry-Perot prediction given by Eq. (7).

The structure of the field in the present case is such that one can regard the grating openings as an ensemble of independent emitters. They are coherently excited by the incident pulse, and their coherent emission builds up the radiation field associated with the resonantly enhanced transmission (reflection) properties of the grating. This is in contrast with the previously reported TM results, where the excitation of plasmons leads to the coupling between effective emitters associated with the grating openings. This point is further illustrated in Fig. 5, where we show the transmission coefficient calculated for the grating structure with parameters $\varepsilon$ $=11.9, a=0.3 \mu \mathrm{m}, h=0.8 \mu \mathrm{m}$, and different periods $D_{g}$ $=D_{0} \equiv 1.75 \mu \mathrm{m}, D_{g}=D_{0} / 1.5$, and $D_{g}=D_{0} / 4$. The change of the grating period does not affect positions of the peaks in the transmission coefficient, pointing at the independence of the trapped field associated with different openings. On the contrary, for the TM-polarized light, the resonant wavelengths are "pinned" to the grating period ${ }^{5,6,1}$ — this fact being a reason for the ongoing discussion on the role of surface plasmons in the TM radiation transmission. The overall increase of the transmission coefficient when the grating period is reduced is due to the increase of the density of emitters (openings), while the time scale of the lasing effect remains the same because it is set by the attenuation of the metal and by the coupling of each individual grating region to the vacuum. In agreement with Eq. (1), a direct comparison of the result for $D_{g}=D_{0}$ with the results presented in Fig.

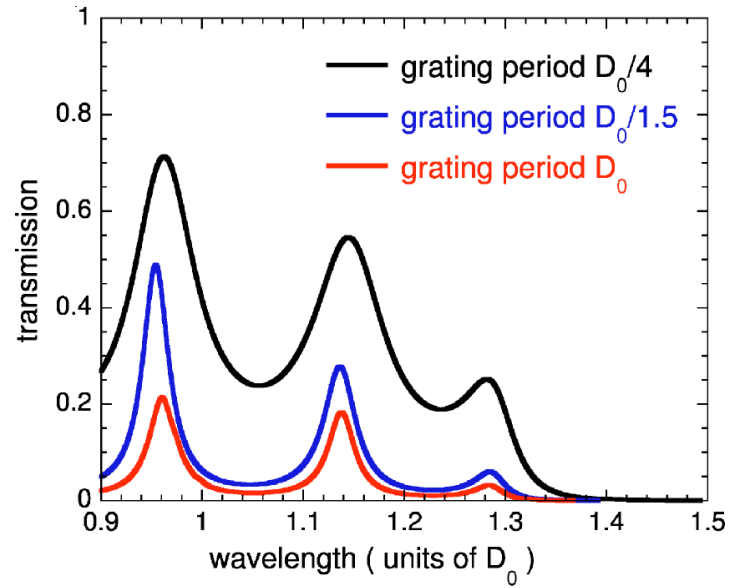

FIG. 5. (Color online) Zero-order transmission coefficient as a function of the wavelength of the incident radiation measured in units of the period $D_{0} \equiv 1.75 \mu \mathrm{m}$. The computed data are given for a grating structure characterized by $\varepsilon=11.9, \quad a=0.3 \mu \mathrm{m}, \quad h$ $=0.8 \mu \mathrm{m}$, and different periods $D_{g}$. The red curve shows the transmission coefficient for $D_{g}=D_{0} \equiv 1.75 \mu \mathrm{m}$, the blue curve for $D_{g}$ $=D_{0} / 1.5$, and the black curve for $D_{g}=D_{0} / 4$.

3 shows that the reduction of $h$, and primarily of $a$, leads to the blueshift of the whole resonance series. A similar dependence of the resonance wavelengths on the grating thickness $h$ has been reported as well for the TM polarization. ${ }^{5,6}$ However, some caution is needed when comparing the TE and $\mathrm{TM}$ results in view of their different boundary conditions.

\section{SIGNIFICANCE OF TRAPPED MODES IN RESONANT SCATTERING}

The example of the grating structure considered above suggests that knowledge of long-lived trapped modes is crucial for a custom design of nanostructured materials with enhanced transmission (reflection) properties in designated narrow intervals of wavelengths. Here we offer a rather general approach which establishes a direct relation between transmission (reflection) properties of nanostructured materials and the existence of trapped modes. We assert that once the Maxwell equations have been reformulated in Schrödinger form, the significance of trapped modes for the light transmission can immediately be understood from the basic principles of quantum resonant scattering theory. ${ }^{35}$

Recall that the approach relies on a representation of the total wave function of the system as a sum of nonresonant and resonant contributions. ${ }^{31,32}$ Consider the case without losses so that the corresponding Hamiltonian is Hermitian. Let $H$ be the total Hamiltonian of a nanostructured material, supporting resonance(s), and $H_{0}$ be a Hamiltonian responsible for a nonresonant scattering. In the present case $H$ and $H_{0}$ are Hamiltonians of the metal grating with and without dielectric insertions, respectively. For the TM polarization the natural choice will be to set $H_{0}$ to be a Hamiltonian of the simple metal slab with no gratings. The LippmannSchwinger formalism ${ }^{33,34}$ is applied to describe the scattering of a plane wave on a scatterer that has resonant excitations. Now we will show that the existence of long-lived quasista- 
tionary states in the symmetric $(z \rightarrow-z$ transformation) grating with dielectric insertions implies that there exists a frequency at which the grating becomes transparent. If $\omega$ is the frequency of the incoming wave, then a solution of the Schrödinger equation $(H-\omega) \Psi=0$ can be written in the form

$$
\Psi=\Psi_{0}+G^{+}(\omega)\left(H-H_{0}\right) \Psi_{0} \equiv \Psi_{0}+\Psi^{+},
$$

where $G^{+}(\omega)=[\omega-H+i \eta]^{-1}, \eta \rightarrow 0+$ is the Green function, $\Psi_{0}$ is a solution of $\left(H_{0}-\omega\right) \Psi_{0}=0$, and $\Psi^{+}$satisfies radiation (outgoing wave) boundary conditions and describes the scattered wave due to dielectric insertions. If the incoming wave is polarized along the grating, then the wave functions $\Psi_{0}$ and $\Psi^{+}$contain only one component of the electric field which is denoted $E_{0}$ and $E^{+}$, respectively. Let the frequency $\omega$ be in the range in which the structure described by $H_{0}$ Hamiltonian is a total reflector; then,

$$
E_{0} \rightarrow A_{0} e^{i k z}+A_{0}^{*} e^{-i k z}, \quad z \rightarrow-\infty,
$$

and $E_{0}$ vanishes as $z \rightarrow \infty$ assuming that the grating is centered at $z=0$. For the TM polarization, the magnetic field should be considered along similar lines.

Quasistationary states, or resonances, that exist in the grating with dielectric insertions correspond to eigenvectors of $H$ with outgoing wave boundary conditions and, therefore, they are associated with poles of the Green function $G^{+}(\omega)$. Note that due to the complex boundary conditions $H$ would have complex eigenvalues $\omega_{0}-i \Gamma / 2$ with negative imaginary parts $(\Gamma>0)$ which specify resonance widths. Thus, in the vicinity of a pole, the frequency dependence of the Green function can be approximated by

$$
G^{+}(\omega) \sim \frac{1}{\omega-\omega_{0}+i \Gamma / 2} .
$$

Let $\omega$ be near a resonant frequency (within the resonance width), while the resonant frequency $\omega_{0}$ is assumed to remain in the range of total reflectivity of the pure metallic grating. In this case, from the symmetry of the Hamiltonian $H$ under the parity transformation, $z \rightarrow-z$, it follows that

$$
E^{+} \rightarrow[\operatorname{sgn}(z)]^{p} A^{+} e^{ \pm i k z}, \quad z \rightarrow \pm \infty,
$$

where the parity factor of $A^{+}$corresponds to either a symmetric $(p=0)$ or antisymmetric $(p=1)$ eigenfunction of $H$. The lowest-frequency resonance corresponds to the symmetric solution. The amplitude $A^{+}$has to be found from energy flux conservation. The incident flux is $\left|A_{0}\right|^{2}$. The outgoing flux is $\left|A_{0} \pm A^{+}\right|^{2}+\left|A^{+}\right|^{2}$. Let $\phi_{0}$ and $\phi^{+}$be the phases of $A_{0}$ and $A^{+}$, respectively. Then from flux conservation we infer that $\left|A^{+}\right|^{2}=\left|A_{0}\right|^{2} \cos ^{2}\left(\phi_{0}-\phi^{+}\right)$. From Eq. (12) it follows that $\phi^{+}$, as a function of the frequency $\omega$, rapidly changes by $\pi$ over a small interval containing the resonant frequency (the eigenvalue of $H$ ), while the phase $\phi_{0}$ describing the nonresonant scattering is nearly constant, or changes slowly. Therefore by continuity of $\phi^{+}$in the vicinity of the resonant frequency there exists a frequency at which $\left|A_{0}\right|^{2}=\left|A^{+}\right|^{2}$; that is, the incoming flux coincides with the transmitted flux and the grating becomes transparent.

Thus, in the absence of attenuation, the existence of trapped electromagnetic mode(s) or resonances necessarily leads to $100 \%$ transmission close to the wavelengths of the trapped modes in symmetric nanostructured materials which otherwise (in the absence of such modes) are not transparent. The same approach can be used to analyze a possible $100 \%$ reflection in dielectric gratings, which otherwise (in the absence of trapped modes) are nearly transparent in the zero diffraction order. It also explains possible Fano profiles in the transmission (reflection) coefficient in the cases when nonresonant scattering described by the Hamiltonian $H_{0}$ leads to both reflection and transmission.

In a generic case, in order for $100 \%$ transmission to be possible, the parity symmetry of the Hamiltonian (system) is not required, but the weaker condition (13) on the asymptotic behavior of $\Psi^{+}$is indeed necessary. The latter readily follows from flux conservation. A sufficient condition for $100 \%$ transmission is the absence of scattering channels with different quantum numbers at the resonance frequency-e.g., higher-order diffraction and/or coupling to scattering states with different polarization. All these effects lead to a breaking of the flux conservation in a selected scattering channel. If the coupling to other channels is not significant, an enhanced (not 100\%) transmission can still be observed in the selected channel. It can be quantified by conventional means of quantum scattering theory applied to the effective electromagnetic Hamiltonian of the system in question. In practice, gratings often have the imperfections which induce a coupling between TE and TM polarization. Thus, in the TE resonant scattering channel $100 \%$ transmission will be lost due to a leaking of the energy flux into higher-order diffraction and/or TM polarization channels induced by the imperfections. One should not, however, expect a resonant transmission in the TM channel facilitated by the imperfections since the resonant (enhanced) transmission is essentially due to the constructive interference which, in turn, occurs thanks to the periodicity of the structure, while imperfections are usually randomly distributed. In fact, in a lossless grating, a deviation of the transmission coefficient from one at the resonant frequency can be used as a measure of the grating quality.

Finally, it is worth mentioning that a loss of the electromagnetic energy in dispersive materials prevents $100 \%$ transmission (reflection) from occurring, again because of breaking of flux conservation even in a single-scattering channel available. This is clearly seen from our numerical results with and without attenuation (Fig. 3). Our formalism offers a possibility to quantify such effects by studying the unitarity violation of the scattering matrix caused by the skew-Hermitian part of the effective Hamiltonian (see, e.g., Ref. 36). Note that in quantum systems a leak of the probability density into scattering channels weakly coupled to the one of interest is often modeled by an effective nonHermitian Hamiltonian for the main (selected) scattering channel(s) only.

\section{CONCLUSIONS}

We have elucidated the role of trapped modes in the extraordinary light transmission in nanostructured materials by reformulating the Maxwell equations for passive linear media in the form of the time dependent Schrödinger equation 
and applying to the latter the basic principles of quantum resonant scattering theory-in particular, the LippmannSchwinger formalism. Trapped electromagnetic modes in nanostructured materials play the same role as resonances in quantum scattering. This offers well-developed quantum mechanical techniques to study resonant light transmission and reflection properties of gratings and other nanostructured materials. We have illustrated this approach by a detailed numerical study of a metal-dielectric grating. In particular, for the TE polarization of the normal incident radiation, the grating, while being a total reflector in the zero diffraction order when no dielectric fillings are present, has been shown to become transparent for certain (resonant) wavelengths when the fillings are present. In accordance with quantum resonant scattering theory, stationary states have been observed in the latter case and none in the former.

\section{ACKNOWLEDGMENTS}

A.G.B. and S.V.S. thank the DIPC for the support and hospitality. Useful discussions with Dr. G. Gómez-Santos are gratefully acknowledged. S.V.S. is grateful to Professor J.R. Klauder for fruitful and stimulating discussions and Dr. R. Albanese and Dr. T. Olson for their continued support.
*Corresponding author. FAX: +33-(0)1-69-15-76-71. Electronic address: borisov@1cam.u-psud.fr

${ }^{1}$ E. Yablonovitch, Phys. Rev. Lett. 58, 2059 (1987).

${ }^{2}$ J. D. Joannopoulos, R. D. Meade, and J. N. Winn, Photonic Crystals (Princeton University Press, Princeton, NJ, 1995).

${ }^{3}$ H. J. Lezec, A. Degiron, E. Devaux, R. A. Linke, L. MartinMoreno, F. J. Garcia-Vidal, and T. W. Ebbesen, Science 297, 820 (2002).

${ }^{4}$ T. W. Ebbesen, H. J. Lezec, H. F. Ghaemi, T. Thio, and P. A. Wolff, Nature (London) 391, 667 (1998).

${ }^{5}$ F. J. Garcia-Vidal and L. Martin-Moreno, Phys. Rev. B 66, 155412 (2002).

${ }^{6}$ J. A. Porto, F. J. Garcia-Vidal, and J. B. Pendry, Phys. Rev. Lett. 83, 2845 (1999).

${ }^{7}$ Y. Takakura, Phys. Rev. Lett. 86, 5601 (2001).

${ }^{8}$ U. Schröter and D. Heitmann, Phys. Rev. B 58, 15419 (1998).

${ }^{9}$ M. M. J. Treacy, Appl. Phys. Lett. 75, 606 (1999).

${ }^{10}$ M. M. J. Treacy, Phys. Rev. B 66, 195105 (2002).

${ }^{11}$ Q. Cao and Ph. Lalanne, Phys. Rev. Lett. 88, 057403 (2002).

${ }^{12}$ P. Lalanne, C. Sauvan, J. P. Hugonin, J. C. Rodier, and P. Chavel, Phys. Rev. B 68, 125404 (2003).

${ }^{13}$ J. M. Steele, C. E. Moran, A. Lee, C. M. Aguirre, and N. J. Halas, Phys. Rev. B 68, 205103 (2003).

${ }^{14}$ S. A. Darmanyan and A. V. Zayats, Phys. Rev. B 67, 035424 (2003).

${ }^{15}$ F. Yang and J. R. Sambles, Phys. Rev. Lett. 89, 063901 (2002).

${ }^{16}$ W.-C. Tan, T. W. Preist, and R. J. Sambles, Phys. Rev. B 62, 11134 (2000).

${ }^{17}$ R. W. Wood, Phys. Rev. 48, 928 (1935).

${ }^{18}$ R. Magnusson and S. S. Wang, Appl. Phys. Lett. 61, 1022 (1992).

${ }^{19}$ S. Peng and G. M. Morris, Opt. Lett. 21, 549 (1996).

${ }^{20}$ T. Peter, R. Bräuer, and O. Bryngdahl, Opt. Commun. 139, 177
(1997).

${ }^{21}$ K. Koshino, Phys. Rev. B 67, 165213 (2003).

${ }^{22}$ L. Pilozzi, A. D’Andera, and R. Del Sole, Phys. Rev. B 54, 10763 (1996)

${ }^{23}$ A. G. Borisov and S. V. Shabanov, J. Comput. Phys. 199, 742 (2004).

${ }^{24}$ A. G. Borisov and S. V. Shabanov (unpublished).

${ }^{25} \mathrm{R}$. G. Newton, Scattering Theory of Waves and Particles (McGraw-Hill, New York, 1966); C. J. Joachain, Quantum Collision Theory (North-Holland, Amsterdam, 1975).

${ }^{26}$ S. V. Shabanov, http://xxx.lanl.gov/abs/math.NA/0312296

${ }^{27}$ D. Kosloff and R. Kosloff, J. Comput. Phys. 52, 35 (1983).

${ }^{28}$ Numerical Grid Methods and Their Application to Schrödinger's Equation, NATO Advanced Study Institute, Series C: Mathematical and Physical Sciences Vol. 412, edited by C. Cerjan (Kluwer Academic, London, 1993).

${ }^{29}$ A. J. H. M. Meijer, E. M. Goldfield, S. K. Gray, and G. G. Balint-Kurti, Chem. Phys. Lett. 293, 270 (1998); J. Sjakste, A. G. Borisov, J. P. Gauyacq, and A. K. Kazansky, J. Phys. B 37, 1593 (2004).

${ }^{30}$ P. G. Petropoulos, J. Appl. Math. Mech. 60, 1037 (2000); J.-L. Vay, J. Comput. Phys. 165, 511 (2000).

${ }^{31}$ U. Fano, Phys. Rev. 124, 1866 (1961).

${ }^{32}$ See detailed discussion in F.-M. Dittes, Phys. Rep. 339, 215 (2000).

${ }^{33}$ B. A. Lippmann and J. Schwinger, Phys. Rev. 79, 469 (1950).

${ }^{34}$ B. A. Lippmann, Phys. Rev. Lett. 16, 135 (1966).

${ }^{35}$ An analogy between the resonant scattering in optics (the FabryPerot modes) and quantum mechanics (quasistationary states) has already been discussed in K. W. McVoy, L. Heller, and M. Bolsterli, Rev. Mod. Phys. 39, 245 (1967).

${ }^{36}$ A. Böhm, Quantum Mechanics: Foundations and Applications (Springer-Verlag, New York, 1993). 